Classification

Physics Abstracts

76.90

\title{
Muon hyperfine fields in rare earth hosts
}

\author{
I. A. Campbell
}

Laboratoire de Physique des Solides, Bât. 510, Université Paris-Sud, Centre d'Orsay, 91405 Orsay, France

(Reçu le 6 septembre 1983, accepté le 15 novembre 1983)

Résumé. - Un muon implanté dans une terre rare créera un gradient de champ électrique local qui agira sur l'orientation des moments voisins avec pour conséquence une modification importante du champ magnétique dipolaire sur le site du muon.

\begin{abstract}
When a muon is implanted in a rare earth host the electric field gradient created by the muon charge will affect the orientation of neighbouring rare earth moments and so will lead to strong dipolar fields at the muon site.
\end{abstract}

The hyperfine field has been measured at positive muons in a number of metallic ferromagnet hosts, in particular in the rare earths Gd and Dy. In Gd the total hyperfine field $B_{\mu}=B_{\text {int }}+B_{\mathrm{L}}$ is $+1.1 \mathrm{kG}$ [1], where $B_{\mathrm{L}}$ is the Lorentz field and $B_{\text {int }}=B_{\mathrm{hf}}+B_{\mathrm{dip}}$ is the internal field, the sum of a Fermi hyperfine field term $B_{\mathrm{hf}}$ and a dipole term $B_{\mathrm{dip}}$. In $\mathrm{Gd} B_{\mathrm{L}}=+7.4 \mathrm{kG}$ and $B_{\text {dip }}=0.66 \mathrm{kG}$ at an octohedral interstitial site so $B_{\mathrm{hf}}=-7.0 \mathrm{kG}$ [1]. For a number of non-magnetic sp impurities in rare earth hosts $B_{\mathrm{hf}}$ is roughly proportional to the rare earth spin polarization [2,3] so we would expect $B_{\mathrm{hf}}$ to be about $-5 \mathrm{kG}$ in Dy. However the experimental value of $B_{\mu}$ in Dy is $\pm 12.3 \mathrm{kG}$ [4] meaning that, after correction for a small dipole field and the Lorentz field, $B_{\mathrm{hf}}$ is $-0.7 \mathrm{kG}$ or $-25.2 \mathrm{kG}$ depending on the sign of $B_{\mu}$. I suggest that the origin of this apparent anomaly lies in the electric field gradient created by the muon charge which can lead to effects analogous to O. Hartmann's muon induced nuclear quadrupole alignment [5].

For systems having pure spin local moments the coupling between electric field gradients and the magnetic moments is weak ; this is the case in $\mathrm{Gd}$ (and also in $\mathrm{Fe}, \mathrm{Co}$ and $\mathrm{Ni}$ ). Then we can ignore the electric field gradient produced by the muon charge and consider that the orientation of the surrounding magnetic moments is the same as if the muon was absent. However for the rare earths other than Gd which have strong orbital moments and associated non-spherical $f$ electron charge distributions, it is well known that electric field gradients are important. For the particular case of Dy, the introduction of a positive charge next to a Dy site will be such as to produce an axial field gradient. Kikkert and Niesen [6] have shown that for an effective point charge of $-|e|$ at near neighbour distance to a Dy impurity in a noble metal host the total overall uniaxial crystal field splitting is of the order of $400 \mathrm{~K}$ (the neighbour charge in this case is due to a vacancy). For a Dy atom near neighbour to a muon in Dy metal the local electric field 
gradient can be expected to be of similar magnitude but of the opposite sign $\left({ }^{1}\right)$. Because the quadrupolar Stevens factor for Dy is negative the effect of the field gradient will be to force the Dy moment into a direction perpendicular to the axis linking the Dy atom and the muon. This field gradient is strong compared with the magnetic interaction $\left(T_{N}=176 \mathrm{~K}\right)$ or the crystal field, so in the ferromagnetic state the local moments next to the muon will to a first approximation lie perpendicular to the ion-muon axis in the direction which lies closest to the overall magnetization direction. This reorientation of the moments surrounding the muons will have a particularly drastic effect on the dipole field seen at the muon site.

We can do an explicit calculation of the local dipole fields, with the following assumptions :

i) the muon occupies an octohedral site,

ii) the axial electric field gradient dominates both the magnetic interaction and the normal crystal field for Dy near neighbours to the muon, and the effective charge on the muon is positive,

iii) only near neighbour terms contribute significantly to the local dipole field on the muon.

We then use the general dipole-dipole interaction expression

$$
E=\frac{\gamma_{1} \gamma_{2} \hbar^{2}}{r_{12}^{3}}\left[\mathbf{I}_{1} \cdot \mathbf{I}_{2}-\frac{3\left(\mathbf{I}_{1} \cdot \mathbf{r}_{12}\right)\left(\mathbf{I}_{2} \cdot \mathbf{r}_{12}\right)}{r_{12}^{2}}\right]
$$

to estimate the local dipole field at the muon site in ferromagnetic Dy. We find $B_{\text {dip }} \simeq-28 \mathrm{kG}$ if the host magnetization lies along the easy $a$ axis (for the magnetization along the $b$ axis, $B_{\text {dip }} \simeq-25 \mathrm{kG}$ ). This high value is clearly an upper limit, as the axial field does not really totally dominate the other terms, but it can be used as an order of magnitude to compare with experiment. It appears reasonable to deduce that the total field $B_{\mu}$ at a muon in Dy is negative, $-12.3 \mathrm{kG}$, made up of a Lorentz term $+12.6 \mathrm{kG}$, a Fermi hyperfine field of about $-5 \mathrm{kG}$, and a local dipole field of about $-20 \mathrm{kG}$, i.e. rather weaker but of the same order of magnitude as the dipole field calculated above.

It is obviously important to verify experimentally if the sign of the total field in Dy is indeed negative. Assuming this is the case, we can also estimate on the same model what fields we would expect in other ferromagnetic rare earth hosts. The values of the dipole fields calculated with the same assumptions as above are given in table I. There should be a dramatic change of local dipole fields between the cases of $\mathrm{Tb}$, Dy and Ho where the quadrupolar Stevens factor is negative, and the cases of Er and Tm where it is positive. For the former the ion moments should tend to lie perpendicular to the muon-ion axis, while for the latter the moments should tend to lie along this axis. In consequence the local dipole field changes sign.

Table I. - Calculated local dipole field in $\mathrm{kG}$ on the muon obtained from the model explained in the text. The axes given here are the possible ferromagnetic moment axes of the rare earths.

$\begin{array}{lccc}\text { Axis } & a & b & c \\ - & - & -\overline{22.5} & - \\ \mathrm{Tb} & -25 & -2.5 & \\ \mathrm{Dy} & -28 & -25.3 & \\ \mathrm{Ho} & -29 & -25.7 & \\ \mathrm{Er} & & & +35 \\ \mathrm{Tm} & & & +27.5\end{array}$

$\left({ }^{1}\right)$ Throughout it will be assumed that the muon charge is not overscreened by the conduction electrons. It is possible that this assumption is incorrect, in which case the signs in table I will be changed, and the detailed predictions will be somewhat different. 
We can now make rough estimates of the total internal field $B_{\mathrm{hf}}+B_{\mathrm{dip}}$ assuming that $B_{\mathrm{hf}}$ is proportional to $S_{z}$ with the constant of proportionality given by the value for $\mathrm{Gd}$, and that $B_{\mathrm{dip}}$ is reduced from the limiting model value by a factor of 1.4 as in Dy (assuming of course that the field in Dy is indeed negative). We find the set of values given in table II, which it would be interesting to check on experimentally. Experiments would need to be made in applied fields on single crystal samples both to obtain the sign of the fields and to induce a simple ferromagnetic state in Ho, Er and Tm.

Table II. - Calculated internal fields at the muon in different rare earths in $\mathrm{kG}$. The assumptions made are given in the text.

$$
\begin{array}{cccccc} 
& \text { Tb } & \text { Dy } & \text { Ho } & \text { Er } & \text { Tm } \\
B_{\text {int }} & -22 & (-25) & -22 & +22 & +17
\end{array}
$$

We have not made estimates of the fields in the antiferromagnetic states of the rare earths as this would involve more complicated and lengthy calculations which are not warrented at this stage.

Although we have only discussed explicitly pure rare earth metal hosts, the same mechanism should be important for muons in all intermetallic compounds and alloys containing rare earths other than Gd. When more experimental values become available it should be possible to refine the assumptions made above. Such experiments should give useful information on the effective screening of the muon charge. Similar but less dramatic effects should also be expected for other interstitial or even substitutional impurities in rare earth hosts, and this mechanism should be considered when discussing hyperfine field data at any dilute impurity in a rare earth metal or compound.

Finally, this mechanism should also tend to break down magnetic order in rare earth or compound hosts where it is possible to dissolve a finite concentration of hydrogen or other interstitial atoms [7].

After this paper was submitted for publication, I learnt that Schillaci et al. [8] had also considered some of the implications of the electric field gradient around the muon.

\section{References}

[1] Kundig, W., Hyp. Int. 9 (1981) 571.

[2] Forker, M. and Hammersfahr, A., Z. Phys. 263 (1973) 33.

[3] Kuchma, A. S. and ShPinel, V. S., J.E.T.P. 35 (1972) 556.

[4] Hofmann, W. et al., Phys. Lett. A 65 (1978) 343.

[5] Hartmann, O., Phys. Rev. Lett. 39 (1977) 832.

[6] Kikkert, P. J. and Niesen, L., Phys. Rev. B 23 (1981) 2126, and to be published.

[7] Daou, J. N., Vajda, P., Lucasson, A. and Lucasson, P., J. Phys. C 14 (1981) 129.

[8] Schillaci, M. E. et al., To be published in the Proceedings of the Yamada Conference on Muon Spin Rotation. 